\title{
Settling and collision between small ice crystals in turbulent flows
}

\author{
Jennifer Jucha, ${ }^{1}$ Aurore Naso, ${ }^{2}$ Emmanuel Lévêque, ${ }^{2}$ and Alain Pumir ${ }^{3}$ \\ ${ }^{1}$ Projektträger Jülich, Forschungszentrum Jülich GmbH, D-52425, Jülich, Germany. \\ ${ }^{2}$ Laboratoire de Mécanique des Fluides et d'Acoustique, \\ CNRS, École Centrale de Lyon, Université de Lyon, INSA de Lyon, \\ 36 avenue Guy de Collongue, 69134 Écully Cedex, France \\ ${ }^{3}$ Laboratoire de Physique, CNRS, École Normale Supérieure de Lyon, Université de Lyon, \\ 46 allée d'Italie, 69364 Lyon Cedex 07, France
}

(Dated: May 10, 2017)

\begin{abstract}
Ice crystals are present in a variety of clouds, at sufficiently low temperature. We consider here mixed-phase clouds which, at temperature $\gtrsim-20^{\circ} \mathrm{C}$, contain ice crystals, shaped approximately as thin oblate ellipsoids. We investigate the motion of these particles transported by an isotropic turbulent flow, and in particular, the collision between these crystals, a key process in the formation of graupels. Using fully-resolved direct numerical simulations, and neglecting the effects of fluid inertia on the particle motion, we determine the influence of the turbulence intensity and of gravitational settling, in a realistic range of parameters. At small Reynolds numbers, collisions are induced mainly by differential gravitational settling between particles with different orientations. The effect has a clear signature on the relative orientation of colliding ellipsoids. As the Reynolds number increases, however, the influence of the turbulent velocity fluctuations becomes the dominant effect determining the collision rate. Using simple estimates, we propose an elementary understanding of the relative importance of gravitational settling and turbulent fluctuations.
\end{abstract}

\section{INTRODUCTION}

The condensation of water vapour in many types of clouds involves both liquid droplets and ice crystals [1]. These droplets and ice crystals control many properties, including optical properties such as reflection of light [2-4]. As such, clouds contribute in an essential way to the energy balance of the earth. The lack of a precise understanding of the structure and composition of clouds induces very significant inaccuracy when estimating quantitatively the effect of clouds on climate, thus causing large uncertainties in any attempts to predict the climate evolution. In clouds, the growth of water droplets or crystals leads to the formation of water drops or graupels, hence to precipitation. Much work has been devoted lately to the formation of rain drops in warm clouds, where the ice phase is totally absent [5-7]. One of the outstanding questions there is to understand the fast formation of rain drops, of size $\sim 1 \mathrm{~mm}$, starting from a population of very small droplets of size $\sim 10 \mu \mathrm{m}$. Overall, a quantitatively accurate description of this phenomenon remains difficult.

We are interested here in the class of "mixed-phased" clouds, where water vapour droplets coexist with ice crystals. This is typically the case in cumulus clouds, in a range of temperature $T$ between $-30^{\circ} \mathrm{C} \lesssim T \lesssim 0^{\circ} \mathrm{C}$. The shape of the crystals depends on the temperature $[1,8]$. Over a range of temperatures $\left(-20^{\circ} \mathrm{C} \lesssim T \lesssim-10^{\circ} \mathrm{C}\right)$, ice crystals are shaped like snow-flakes (they are columnar shaped at lower temperature, or in the range $-10^{\circ} \mathrm{C} \lesssim T \lesssim-5^{\circ} \mathrm{C}[1,8,9]$ ). As a simplified assumption, the snow-flake shaped ice crystals can be approximated as very thin oblate ellipsoids, with an aspect ratio $\beta$ between the short and the long axes, respectively equal to $c$ and $a(\beta=c / a)$ in the range: $0.01 \lesssim \beta \lesssim 0.05$.

Gravitational settling is known to play a significant role in several different contexts in cloud physics [10, 11], including the problem of gravitational coagulations [1]. In the case of clouds containing only very small droplets (no ice), droplets of size of the order of $\sim 10 \mu \mathrm{m}$, nucleated from aerosols, need to grow, essentially by aggregation with other small droplets, to form objects that will lead to precipitation. Larger particles settle faster than smaller ones, thus creating a very strong mechanism to generate collisions. In a monodisperse suspension of droplets, which is the case shortly after nucleations, the gravitational mechanism is not expected to play much of a role. Whether the stirring by turbulence is efficient enough to generate a large enough number of collisions between small droplets flow remains open $[5,7,12]$. Studies aimed at quantifying the role of turbulence and settling for a monodisperse solution of (spherical) droplets have shown that at a fixed turbulence intensity, collision rates decrease when increasing gravitational settling $[13,14]$.

The situation is noticeably different in the presence of ice crystals. In fact, in still air, the settling velocity of a crystal (or equivalently for the present purpose, of an oblate ellipsoid) depends on the orientation of the crystal with respect to gravity, g. This creates a dispersion of the settling velocity, which may greatly enhance the collision rate [15]. One of the main findings of our study is that this mechanism plays a very important role at low turbulence intensity, and certainly affects the distribution of relative orientations when ice crystals collide. This effect of differential settling 
velocity, however, becomes much weaker at higher turbulence intensity.

Our work is based on direct numerical simulations (DNS) of homogeneous and isotropic turbulence, using pseudospectral methods. The calculation is forced at low wavenumbers, in a periodic box of size $8 \pi \mathrm{cm} \approx 25 \mathrm{~cm}$, with an energy dissipation rate $\varepsilon$ in the range $1 \mathrm{~cm}^{2} / \mathrm{s}^{3}-256 \mathrm{~cm}^{2} / \mathrm{s}^{3}$. We stress that the need to extrapolate the velocity gradient tensor to determine the motion and orientation of ice crystals led us to choose a very high resolution of the turbulent flow. Whereas our choice of a simplified turbulent flow potentially leaves aside important aspects of the flow in a cloud, it allows us to obtain accurate numerical data, and to improve our understanding of the various physical effects affecting the collision rate. The intensity of turbulence is often characterized by the Reynolds number based on the Taylor micro scale, $R_{\lambda}$. In our setup, with a fixed system size and viscosity, the relation between $R_{\lambda}$ and $\varepsilon$ is one-to-one. In our simulations, $R_{\lambda}$ varies from $\approx 56$ to 151 . In the flow, we simulate the motion of oblate ellipsoids of long axis $a=150 \mu \mathrm{m}$ and of short axis $c=\beta a, \beta=0.02$. We also varied $\beta$ at an intermediate Reynolds number corresponding to $\varepsilon \approx 16 \mathrm{~cm}^{2} / \mathrm{s}^{3}(\beta=0.01$ and $\beta=0.05)$. The equations describing the motion of the very small ellipsoids are obtained by neglecting all effects of inertia of the fluid, that is, by using the assumption that the local Reynolds number is very small, so the Stokes equations can be used [16, 17]. Our approach relies on a one-way coupling approach, which ignores the feedback induced by the motion of the particles on the fluid motion. We then investigate the statistical properties of the population of ellipsoids, as well as their collision rate, using the often used "ghost-collision" approximation. Namely, we merely detect when two ellipsoids are in contact. To understand the role of gravitational settling, we conducted simulations both with and without gravity. Our results are organized as follows. The numerical methods used for integrating the flow and the particles dynamics are first described in Section II. We then study in Section III the statistics of orientation and of settling of the ellipsoids. The collisions statistics of these objects are presented and discussed in Section IV. Concluding remarks are finally given in Section V.

\section{NUMERICAL METHODS}

\section{A. Turbulent flows}

The work is based on DNS of homogeneous, isotropic flows, at moderate Reynolds numbers. Density stratification, which would play an important role in clouds, is neglected here. Briefly, the Navier-Stokes equations:

$$
\begin{aligned}
& \partial_{t} \mathbf{u}+(\mathbf{u} \cdot \nabla) \mathbf{u}=-\frac{\nabla p}{\rho_{f}}+\nu \nabla^{2} \mathbf{u}+\mathbf{f} \\
& \nabla \cdot \mathbf{u}=0
\end{aligned}
$$

where $\mathbf{u}$ and $p$ respectively denote the velocity and pressure fields, $\nu$ is the kinematic viscosity of the fluid and $\rho_{f}$ is its density. The Navier-Stokes equations are solved using pseudo-spectral methods in a triply periodic domain of size $(2 \pi)^{3}$. The flow is maintained statistically stationary by forcing the velocity field by a forcing term, $\mathbf{f}$, acting on the low wave-numbers of the flow, and chosen so that the injected power $(\mathbf{f} \cdot \mathbf{u})$, averaged over the entire volume, is equal to $\varepsilon$. The flow is fully dealiased, using the $2 / 3$-rule. Specifically, if $N$ is the number of grid points in each direction, the nonlinear term is computed by using only Fourier modes $0 \leq n \leq N / 3$. The number of points taken here are $N=384$, 768 and 1536 . The energy dissipation rate $\varepsilon$ has been adjusted so that $k_{\max } \eta \gtrsim \pi, \eta$ being the characteristic size of the smallest eddies, known as the Kolmogorov scale $\eta=\left(\nu^{3} / \varepsilon\right)^{1 / 4}$, and $k_{\max }=N / 3$ being the highest wavenumber represented. With our resolution, the velocity gradients can be faithfully interpolated at the position of the ellipsoids. The numerical integration rests on a second-order accurate in time Runge-Kutta scheme.

The simulated turbulent flows can be characterized by their Reynolds number based on the Taylor microscale, $R_{\lambda}=u_{r m s} \lambda / \nu$, with $\lambda \equiv \sqrt{15 u_{r m s}^{2} \nu / \varepsilon}$ and $u_{r m s}^{2}=\left\langle u_{x}^{2}\right\rangle\left(u_{x}\right.$ is the $x$-component of the fluid velocity, which is equivalent to any other component by isotropy of the flow). In our cases, $R_{\lambda}=56,95$ and 151 for the three resolutions we used. Alternatively, in an attempt to simulate realistic flow conditions, we used physical units, with the correspondance that the box size is $8 \pi \mathrm{cm}$, the viscosity $\nu=0.1132 \mathrm{~cm}^{2} / \mathrm{s}$, corresponding to cloud conditions [1], and the energy dissipation equal to $0.976 \mathrm{~cm}^{2} / \mathrm{s}^{3}, 15.62 \mathrm{~cm}^{2} / \mathrm{s}^{3}$ and $246.4 \mathrm{~cm}^{2} / \mathrm{s}^{3}$. Given the fact that our forcing imposes an approximately fixed correlation length for the velocity field, and that the viscosity is chosen to be constant, the Reynolds number $R_{\lambda}$ grows essentially as $R_{\lambda} \propto \varepsilon^{1 / 6}$.

\section{B. Ellipsoid dynamics}

In an attempt to simulate particles in realistic conditions, we have fixed various physical constants in a range relevant to the dynamics of ice crystals in clouds, see Table I. 


\begin{tabular}{|c|c|c|c|c|}
\hline \multicolumn{2}{|c|}{ Fluid } & \multicolumn{2}{|c|}{ Particles } & gravity \\
\hline$\rho_{f}, \mathrm{~g} / \mathrm{cm}^{3}$ & $\nu, \mathrm{cm}^{2} / \mathrm{s}$ & $\rho_{p}, \mathrm{~g} / \mathrm{cm}^{3}$ & $a, \mathrm{~cm}$ & $\mathrm{~g}, \mathrm{~cm} / \mathrm{s}^{2}$ \\
\hline $1.41310^{-3}$ & 0.1132 & 0.9194 & 0.015 & 981 \\
\hline
\end{tabular}

TABLE I. Values of the physical parameters used in the simulation. The fluid consists of moist air, whose volumic mass and viscosity are $\rho_{f}$ and $\nu$. The ice particles have a volumic mass $\rho_{p}$, and a major axis $a$. Lastly, the gravity magnitude is $g$.

\section{Equations of motion}

The equations of motion of the ellipsoids are derived by using the expressions of the force and of the torque acting on them by neglecting fluid inertia, that is, by assuming the Reynolds number based on the difference between the velocity of the fluid, $\mathbf{u}$, and that of the particle, $\mathbf{v}$, to be very small. Specifically, solving the hydrodynamics in the low Reynolds number limit, the equation for the translational motion of the center of mass of the ellipsoids reduces to [17]:

$$
\frac{d \mathbf{v}}{d t}=\mathbf{g}+\frac{\nu \rho_{f}}{m_{c}} \mathbf{R}^{-1} \hat{\mathbf{K}} \mathbf{R} \cdot(\mathbf{u}-\mathbf{v})
$$

where $m_{c}=4 \rho_{p} \pi a^{3} \beta / 3$ is the mass of the crystal ( $\rho_{p}$ is the particle density). In Eq. (3), $\mathbf{R}$ is the rotation matrix between the laboratory coordinate system, $(x, y, z)$, and a particle fixed reference system, $(\hat{x}, \hat{y}, \hat{z})$, chosen in such a way that $\hat{x}$ and $\hat{y}$ correspond to the elongated directions of the ellipsoid, and $\hat{z}$ to its short direction. In this frame, the resistance tensor $\hat{\mathbf{K}}$ has a simple diagonal form:

$$
\hat{\mathbf{K}}=16 \pi a^{3} \beta \operatorname{diag}\left(1 /\left(\chi_{0}+a^{2} \alpha_{0}\right), 1 /\left(\chi_{0}+a^{2} \alpha_{0}\right), 1 /\left(\chi_{0}+a^{2} \beta^{2} \gamma_{0}\right)\right) \text {. }
$$

The values of the coefficients are $\alpha_{0}=\left[\beta^{2} /\left(\beta^{2}-1\right)+\arccos (\beta) \beta /\left(1-\beta^{2}\right)^{3 / 2}\right], \gamma_{0}=\left[-2 /\left(\beta^{2}-1\right)-2 \arccos (\beta) \beta /(1-\right.$ $\left.\left.\beta^{2}\right)^{3 / 2}\right]$, and $\chi_{0}=\left[2 a^{2} \beta \arccos (\beta) / \sqrt{1-\beta^{2}}\right]$.

As the ellipsoids are subject to a torque by the action of the fluid [16], they rotate with an angular velocity $\boldsymbol{\Omega} \equiv\left(\Omega_{x}, \Omega_{y}, \Omega_{z}\right)$ in the frame of reference fixed with respect to the particle. The equation of motion for $\Omega$ is obtained by equating the rate of change of angular momentum with torque, in the reference frame of the particle:

$$
\frac{d}{d t}\left(\begin{array}{c}
\Omega_{x} \\
\Omega_{y} \\
\Omega_{z}
\end{array}\right)=\left(\begin{array}{c}
\Omega_{y} \Omega_{z} \frac{\beta^{2}-1}{1+\beta^{2}} \\
\Omega_{z} \Omega_{x} \frac{1-\beta^{2}}{1+\beta^{2}} \\
0
\end{array}\right)+20 \frac{\rho_{f}}{\rho_{p}} \frac{\nu}{a^{2}}\left(\begin{array}{ccc}
\frac{1}{\alpha_{0}+\beta^{2} \gamma_{0}} & 0 & 0 \\
0 & \frac{1}{\alpha_{0}+\beta^{2} \gamma_{0}} & 0 \\
0 & 0 & \frac{1}{2 \alpha_{0}}
\end{array}\right)\left(\begin{array}{c}
\frac{1-\beta^{2}}{1+\beta^{2}} \hat{S}_{y z}+\left(\hat{\Omega}_{z y}-\Omega_{x}\right) \\
\frac{\beta^{2}-1}{1+\beta^{2}} \hat{S}_{x z}+\left(\hat{\Omega}_{x z}-\Omega_{y}\right) \\
\left(\hat{\Omega}_{y x}-\Omega_{z}\right)
\end{array}\right)
$$

where $\hat{\mathbf{S}}$ and $\hat{\boldsymbol{\Omega}}$ are the rate of strain and rate of rotation tensors in the reference frame of the particle, respectively defined as the symmetric and antisymmetric parts of the velocity gradient tensor in the same frame, $\hat{\mathbf{A}}=\mathbf{R} \mathbf{A} \mathbf{R}^{-1}$ $\left(A_{i j}=\partial_{j} u_{i}\right): \hat{\mathbf{S}}=\left(\hat{\mathbf{A}}+\hat{\mathbf{A}}^{t}\right) / 2, \hat{\mathbf{\Omega}}=\left(\hat{\mathbf{A}}-\hat{\mathbf{A}}^{t}\right) / 2$. The rate of rotation of the reference frame, $\boldsymbol{\Omega}$, allows us to determine the rotation matrix $\mathbf{R}$ from the particle coordinate system to the laboratory frame.

The equations of motion (3) and (5) have been implemented in the code, and the numerical results have been carefully compared with those obtained, in the case of simple flows, using Mathematica. In particular, we used a still flow $(\mathbf{u}=\mathbf{0})$ or elementary flows with a few Fourier series modes; on all these flows, we made sure that the solutions were identical to machine accuracy.

\section{Equations of motion in the limiting case of very thin ellipsoids}

As we are interested here mainly in very small values of $\beta$, the resistance tensor $\hat{\mathbf{K}}$ in Eq. $(4)$ reduces to: $\hat{\mathbf{K}} \approx$ $32 a \operatorname{diag}(1 / 3,1 / 3,1 / 2)$. As a consequence, the equation of motion (3) can be then simply expressed in terms of the response time $\tau_{S p}$ of a sphere of radius $a$ in the flow:

$$
\frac{d \mathbf{v}}{d t}=\mathbf{g}+\frac{16}{3 \pi \beta \tau_{S p}} \mathbf{R}^{-1}\left(\begin{array}{ccc}
1 / 3 & 0 & 0 \\
0 & 1 / 3 & 0 \\
0 & 0 & 1 / 2
\end{array}\right) \mathbf{R} \cdot(\mathbf{u}-\mathbf{v})
$$




\begin{tabular}{|c||c|c|c|c|c|c|c|c|c|c|c|c|c|}
\hline & $\varepsilon\left(\mathrm{cm}^{2} / \mathrm{s}^{3}\right)$ & $R_{\lambda}$ & $\beta$ & $\tau_{K}(\mathrm{~s})$ & $T_{L}(\mathrm{~s})$ & $T_{\text {run }}(\mathrm{s})$ & gravity & $N_{\text {ell }}$ & $u_{\text {rms }}(\mathrm{cm} / \mathrm{s})$ & $U_{\text {sett }}(\mathrm{cm} / \mathrm{s})$ & $S t$ & $F r^{s}$ & $F r^{L}$ \\
\hline \hline Run 1 & 0.976 & 55.8 & 0.02 & 0.341 & 1.96 & 59.6 & $\mathrm{Y}$ & $70^{3}$ & 0.546 & 9.78 & 0.034 & 19.6 & 20.7 \\
\hline Run 2 & 0.976 & 55.8 & 0.02 & 0.341 & 1.96 & 59.6 & $\mathrm{~N}$ & $100^{3}$ & 0.546 & 0 & 0.034 & 0 & 0 \\
\hline Run 3 & 15.62 & 94.6 & 0.02 & 0.085 & 0.696 & 14.6 & $\mathrm{Y}$ & $100^{3}$ & 1.43 & 9.60 & 0.135 & 9.8 & 7.9 \\
\hline Run 4 & 15.62 & 94.6 & 0.02 & 0.085 & 0.696 & 14.9 & $\mathrm{~N}$ & $100^{3}$ & 1.43 & 0 & 0.135 & 0 & 0 \\
\hline Run 5 & 15.62 & 94.6 & 0.01 & 0.085 & 0.696 & 14.6 & $\mathrm{Y}$ & $70^{3}$ & 1.43 & 4.88 & 0.068 & 4.9 & 3.95 \\
\hline Run 6 & 15.62 & 94.6 & 0.01 & 0.085 & 0.696 & 14.9 & $\mathrm{~N}$ & $100^{3}$ & 1.43 & 0 & 0.068 & 0 & 0 \\
\hline Run 7 & 15.62 & 94.6 & 0.05 & 0.085 & 0.696 & 14.6 & $\mathrm{Y}$ & $70^{3}$ & 1.43 & 23.44 & 0.34 & 25 & 20.2 \\
\hline Run 8 & 15.62 & 94.6 & 0.05 & 0.085 & 0.696 & 14.9 & $\mathrm{~N}$ & $100^{3}$ & 1.43 & 0 & 0.34 & 0 & 0 \\
\hline Run 9 & 246.4 & 151.2 & 0.02 & 0.021 & 0.265 & 3.69 & $\mathrm{Y}$ & $70^{3}$ & 3.59 & 10.08 & 0.54 & 1.23 & 0.79 \\
\hline Run 10 & 246.4 & 151.2 & 0.02 & 0.021 & 0.265 & 4.11 & $\mathrm{~N}$ & $100^{3}$ & 3.59 & 0 & 0.54 & 0 & 0 \\
\hline
\end{tabular}

TABLE II. Characteristics of the runs discussed here. $T_{L}$ and $\tau_{K}$ respectively denote the integral and Kolmogorov time scales. $T_{\text {run }}$ refers to the time over which collision statistics have been computed. The number of ellipsoids used to estimate the statistical properties is $N_{e l l}$; the root-mean-square of the turbulent velocity fluctuations is $u_{r m s}$, and the settling velocity $U_{s e t t}$ (in runs with $\mathrm{g}$, i.e. with a ' $\mathrm{Y}$ ' in the 'gravity' column).

where

$$
\tau_{S p}=\frac{2 a^{2}}{9 \nu} \frac{\rho_{p}}{\rho_{f}} .
$$

From Eq. (6), the characteristic time of the translational dynamics is of the order of $\beta \tau_{S p}$. Similarly, the expressions $\alpha_{0} \approx \pi \beta / 2$ and $\gamma_{0} \approx 2$, valid when $\beta \rightarrow 0$ reduce the matrix $\operatorname{diag}\left(1 /\left(\alpha_{0}+\beta^{2} \gamma_{0}\right), 1 /\left(\alpha_{0}+\beta^{2} \gamma_{0}\right), 1 /\left(2 \alpha_{0}\right)\right)$ in Eq. (5) to: $\frac{2}{\pi \beta} \operatorname{diag}(1,1,1 / 2)$. This implies that the characteristic time scale of the evolution of $\boldsymbol{\Omega}$ is $\sim \beta \tau_{S p}$.

In physical terms, we chose the size of the ellipsoids to be $a=150 \mu m$, with an aspect ratio equal to $\beta=0.02$ for most of the runs. We also explored the influence of $\beta$, in a range of values relevant to mixed-phase clouds, by considering $\beta=0.01$ and $\beta=0.05$ at $\varepsilon \approx 16 \mathrm{~cm}^{2} / \mathrm{s}^{3}\left(R_{\lambda}=95\right)$. The number of ellipsoids followed during the run is $N_{e l l}$. The characteristics of the runs discussed in this work are summarized in Table II.

\section{Equations of motion: dimensionless numbers}

In the case of spherical particles, it is customary to compare the effects of turbulence, gravitational settling and particle inertia by introducing dimensionless numbers. Namely, the particle relaxation time can be inferred from (6) to be $\tau_{p} \approx 2 \beta \tau_{S p}$. Comparing this time scale to the Kolmogorov time scale leads naturally to the definition of the Stokes number:

$$
S t \equiv 2 \beta \frac{\tau_{S p}}{\tau_{K}} .
$$

Table II shows that the Stokes number varies from values $\approx 0.03$ up to values $\approx 0.5$. Interestingly, in the related problem of collisions between spherical particles, inertial effects become important for $S t \approx 0.4$ i.e. in the range covered by the present study.

To characterize the relative importance of gravitational settling and turbulence, the Froude number is defined, as often done in the context of cloud physics [13], as the ratio between the settling velocity, of order $g \tau_{p}$ and the velocity differences at the Kolmogorov scale $(\varepsilon \nu)^{1 / 4}$. Using (6), we introduce

$$
F r^{s} \equiv 2 \beta \frac{g \tau_{S p}}{(\nu \varepsilon)^{1 / 4}}
$$

As indicated in Table II, for all the runs in the presence of gravitational settling, the Froude number is $F r^{s} \gtrsim 1$.

Whether the values of $\mathrm{Fr}^{s} \gtrsim 1$ imply that gravity plays such an important role is however not guaranteed. In fact, recent work [18] demonstrates that the distribution of orientation depends on the Stokes number and on $F r / R_{\lambda}^{1 / 2}$. In turbulent flows, the ratio between the rms velocity, $\left\langle u^{2}\right\rangle^{1 / 2}$ and the velocity scale at the Kolmogorov scale, $(\nu \varepsilon)^{1 / 4}$, is 
$\propto R_{\lambda}^{1 / 2}$, which indicates that the settling velocity $g \tau_{S p}$ should be compared with $\left\langle u^{2}\right\rangle^{1 / 2}$. This leads us to define the Froude number $F r^{L}$, based on the large scale velocity of the flow:

$$
F r^{L} \equiv 2 \beta \frac{g \tau_{S p}}{\left\langle u^{2}\right\rangle^{1 / 2}}
$$

The values of $F r^{L}$ are indicated in Table II; the bias of the distribution of $n_{z}$ is clearly prominent for $F r^{L}>1$, see [18] and Section III A. This is an important aspect, since, as demonstrated in this work, the importance of gravitational settling comes mostly from the difference in the settling velocity induced by the differences in the orientations of the ellipsoids.

\section{Limitations of our approach}

The effect of the settling induces a systematic motion of the particles with respect to the turbulent flow. In this context, the use of periodic boundary conditions in the vertical direction may become problematic if the settling becomes very fast, so heavy particles go through the periodicity box in a time which is shorter than the turbulence decorrelation time. Specifically, let $U_{\text {sett }}$ be the average settling velocity $\left(U_{\text {sett }}=-\left\langle v_{z}\right\rangle\right)$, and $T_{\text {sett }} \approx L / U_{\text {sett }}$ the characteristic time of settling of the particles through the entire box. Provided $T_{\text {sett }}$ is large compared to the decorrelation time of the turbulent velocity field $T_{L}$, however, the particle is submitted to a velocity field that significantly differs from that at earlier time. We observe that the value of the settling velocity measured for $\beta=0.05$ and $\varepsilon \approx 16 \mathrm{~cm}^{2} / \mathrm{s}^{3}$ is $U_{\text {sett }} \approx 23.44 \mathrm{~cm} / \mathrm{s}$, so particles settle in a time of $\approx 1 \mathrm{~s}$ through the computational box $L \approx 25.1 \mathrm{~cm}$. This time is larger than the decorrelation time of the velocity field by a factor $\approx 2$, which guarantees that the use of periodic boundary conditions in the vertical direction does not lead to spurious effects.

We end this subsection by recalling that the description of the motion of the ellipsoids using the Stokes equations is only a simplifying approximation. The corrections due to a nonzero Reynolds number of the particle have been the subject of several investigations in the past $[19,20]$. Taking into account finite Reynolds number effects may be particularly important, in particular for the description of the rotational degrees of freedom [21-23]. We note that the torque acting on oblate ellipsoids, and due to finite Reynolds number effects (deviation from the Stokes regime), tends to stabilize the configuration where the ellipsoid settles with its basis horizontal $[1,19,24]$. How the effects of inertia would affect the equation of motion for the angular degrees of freedom is unknown. In the present work, we simply ignore these effects. This is certainly a limitation of the present approach.

\section{Collision Detection}

Collisions are treated here in the so-called "ghost collision" approximation. Namely, we simply follow the motion of ellipsoids in the flow, and merely detect when two ellipsoids are in contact, without modifying their subsequent motion. The method, used in many numerical studies, has been thoroughly tested in the case of spherical particles [25].

Collision detection between particles of non-spherical shapes requires a special approach. We follow here the one proposed by Siewert and collaborators [15, 26]. The method of detection is based on the work of Choi et al. [27]. Specifically, for each ellipsoid, one expresses the equation of the surface in the form: $\mathbf{X} \cdot \mathbf{B}_{i} \cdot \mathbf{X}=1$, where $\mathbf{X}$ is a vector in 4-dimensions: $\mathbf{X}=(x, y, z, 1)$, and the matrix $\mathbf{B}_{i}$ characterizes the $i^{\text {th }}$ ellipsoid. To detect possible contacts between ellipsoids $i$ and $j$, one computes the fourth-order polynomial: $P_{i j}(\lambda)=\operatorname{det}\left(\mathbf{B}_{i}-\lambda \mathbf{B}_{j}\right)$. It is known [27] that the polynomial $P_{i j}$ has exactly two positive, real roots. Whether the two ellipsoids $i$ and $j$ are in contact can be determined from the nature of the two other roots of $P_{i j}$, that can be real (negative) or complex. In the former case, the two ellipsoids are separated, in the latter they overlap. When they are in contact, the polynomial $P_{i j}$ has a real, negative double root. The algorithm implemented for collision detection consists in representing, for each pair of ellipsoids, the evolution of the polynomial $P_{i j}$ as a function both of $\lambda$ and of $t$, and in detecting the formation of a negative double root of $P_{i j}$.

\section{Data analysis}

We followed a set of $70^{3}$ (respectively $100^{3}$ ) ellipsoids in a turbulent flow in the presence (respectively absence) of gravitational settling. Before processing the ellipsoids for collision detection, we let them equilibrate in the flow for at least $\sim \tau_{K}$, where $\tau_{K} \equiv(\nu / \varepsilon)^{1 / 2}$ is the Kolmogorov time. We checked that this is largely sufficient to ensure that the statistical properties of the ellipsoids become stationary. We subsequently followed the motion of the ellipsoids for at 
least $170 \tau_{K}$, or equivalently, 14 times $T_{L}$, the correlation time of the velocity field.

To check the quality of the statistics, we divided the runs into shorter ones, with time intervals of length $\approx 25 \tau_{K}$, and determined the collision rates based on these shorter runs. The collision rates determined from these differed by less than a few percents from the collision rates reported here. Overall, the statistics presented in Section IV were determined with at least 72,000 recorded collisions.

\section{ORIENTATION AND SETTLING STATISTICS OF ELLIPSOIDS IN TURBULENT FLOWS}

From a geometric point of view, the oblate ellipsoids considered in this study can be characterized by the angle between the gravity, $\mathbf{g}$, and the short eigendirection of the ellipsoid, $\mathbf{n}$. We define the unit vector $\mathbf{e}_{z}$ of the (fixed) frame of reference as $\mathbf{e}_{z}=-\mathbf{g} /|\mathbf{g}|$. As represented in Fig. 1a, the cosine between $\mathbf{n}$ and $\mathbf{g}$, or equivalently, $\mathbf{e}_{z}$, therefore reduces to $n_{z}$, up to an immaterial sign. We chose the sign of $n_{z}$, by convention, to be positive throughout.

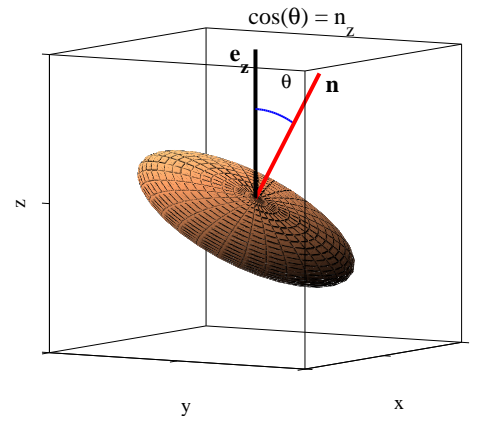

(a)

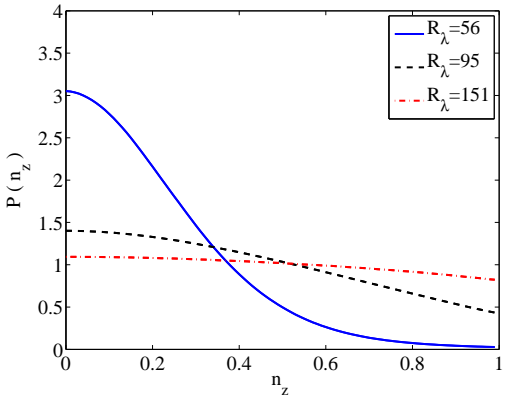

(b)

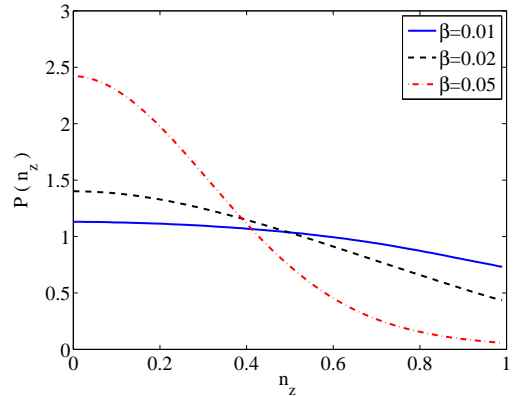

(c)

FIG. 1. (a): Illustration of the definition of the vector $\mathbf{n}$ parallel to the small axis of the ellipsoid. The angle $\theta$ is the angle between $\mathbf{n}$ and the direction of gravity, $\mathbf{g}$. With our definition, $\cos (\theta)=n_{z}$. We restrict ourselves to $0 \leq n_{z} \leq 1$. (b): Distribution of $n_{z}$ for ellipsoids with aspect ratio $\beta=0.02$ in the presence of gravity in a turbulent flow at $R_{\lambda}=56$ (full line), $R_{\lambda}=95$ (dashed line) and $R_{\lambda}=151$ (dashed-dotted line). (c) Distribution of $n_{z}$ in the presence of gravity, at $R_{\lambda}=95$, for ellipsoids of aspect ratio $\beta=0.01$ (full line), $\beta=0.02$ (dashed line) and $\beta=0.05$ (dashed-dotted line).

\section{A. Orientation statistics}

In this subsection, we focus on the distribution of the orientation of the ellipsoid with respect to $\mathrm{g}$, which is relevant not only to the study of collisions, but also to understand the reflection properties of light by clouds loaded with ice crystal $[4,28]$.

The results presented in this subsection are generally in agreement with those of [26], and can be understood theoretically with the help of the formalism developed in [29]. This theoretical approach leads to a quantitative description of the probability distribution functions (PDF) [18]. In the following, we briefly discuss the distribution of $n_{z}$ for ellipsoids with an aspect ratio $\beta=0.02$, at the three values of $\varepsilon$ simulated, and as a function of $\beta$, at $\varepsilon \approx 16 \mathrm{~cm}^{2} / \mathrm{s}^{3}$.

Figure $1 \mathrm{~b}$ shows the distribution of $n_{z}$ for ellipsoids of aspect ratio $\beta=0.02$, at the three Reynolds numbers considered here, in the presence of gravity. In contrast, the distribution of $n_{z}$ is uniformly distributed in the absence of gravity.

At low Reynolds number, the probability distribution is strongly biased towards small values of $n_{z}$, corresponding to ellipsoids settling edge first, which in effect minimizes the drag in the vertical direction. The effect strongly diminishes when the Reynolds number increases, as demonstrated in Fig. 1b: at the highest Reynolds number simulated in this work, $R_{\lambda}=151$, the angles $n_{z}$ are almost uniformly distributed; the corresponding curve differs from 1 by no more than $10 \%$. The observed tendency to relax towards a uniform distribution of $n_{z}$ when the intensity of turbulence, i.e., the energy dissipated in the fluid $\varepsilon$ increases, can be qualitatively understood from the increase of the torque exerted by the turbulent flow on the particles, see Eq. (5).

Figure 1c shows the distribution of orientation of the ellipsoids settling in a turbulent flow at intermediate Reynolds number ( $\varepsilon \approx 16 \mathrm{~cm}^{2} / \mathrm{s}^{3}, R_{\lambda}=96$ ). The main observation is that the ellipsoids with the highest values of $\beta$ are those 
whose distribution of $n_{z}$ is most biased towards small values, see the dashed-dotted line in Fig. 1c. In contrast, for ellipsoids with the smallest value of $\beta$, the distribution of $n_{z}$ is almost uniform, see the full line in Fig. 1c. The trend shown in Fig. 1c can be qualitatively understood by noticing that the mass and the inertia of the ellipsoids increase with $\beta$. Therefore, at a given turbulence intensity, the randomizing effect of the flow is felt more strongly when $\beta$ becomes smaller. In this sense, the trends observed in panels $\mathrm{b}$ and $\mathrm{c}$ of Fig. 1 both illustrate that turbulence tends to randomize the direction of the ellipsoids, the more so as the Reynolds number of the turbulent flow increases, and as the inertia of the ellipsoids decreases.

The conclusions based on the simplified model used here, which completely neglects the corrections due to inertia, contrast with the prediction of a model, based on the existence of a torque, which appears as a correction due to inertia at the lowest order in a Reynolds number expansion, and which favors $n_{z} \approx 1[1,24]$. This may be a limitation of the present work, although the lack of a set of equations taking systematically into account the corrrections due to inertia, even at the lowest in the Reynolds number, makes it difficult to analyze the problem completely (see [18] for a more complete discussion).

We conclude this subsection by pointing out that our study leaves aside interesting properties concerning the correlation between the orientation of the ellipsoids, $\mathbf{n}$, and the velocity gradient tensor or the tumbling rate $\left\langle\dot{\mathbf{n}}^{2}\right\rangle$. These have been recently studied in various cases, mostly when the angular dynamics is overdamped and in the absence of gravity [30-35].

\section{B. Settling velocity}

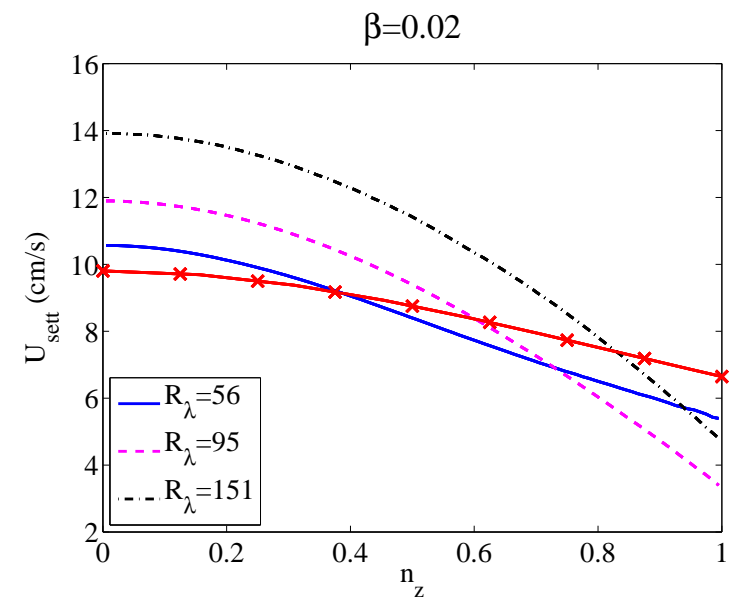

(a)

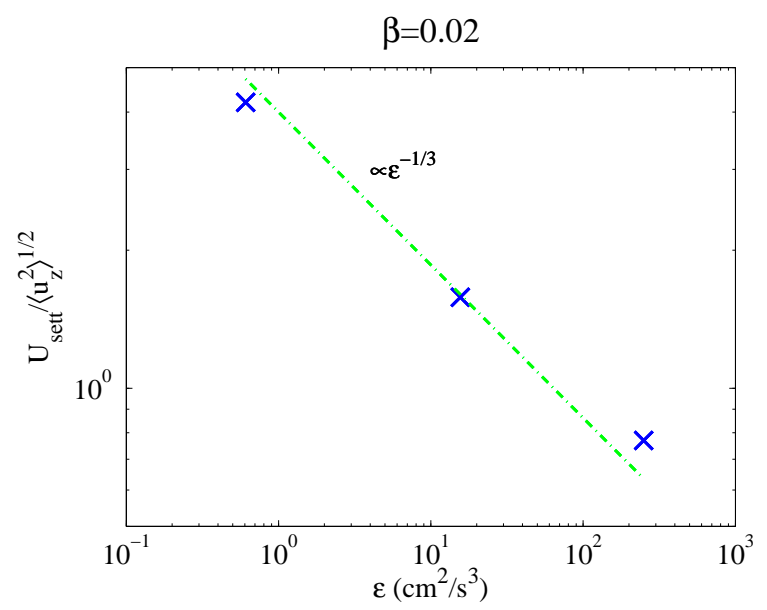

(b)

FIG. 2. Averaged settling velocity of ellipsoids in turbulent flows: (a) settling velocity as a function of $n_{z}$ for ellipsoids with an aspect ratio $\beta=0.02$, at several values of $R_{\lambda}$, as indicated in the legend (for comparison, the settling velocity in still air is shown by the curve with crosses); (b) The ratio between the settling velocity and the rms of the fluctuations. This ratio, which is very close to the Froude number $F r^{L}$ defined by Eq. (10), is shown for $\beta=0.02$, as a function of $\varepsilon$. The dashed line indicates the $\varepsilon^{-1 / 3}$ dependence, which captures qualitatively the dependence obtained numerically.

In a still flow, the settling velocity depends on the value of $n_{z}$. The dependence of the settling velocity of the ellipsoids (absolute value of $\left\langle v_{z}\right\rangle$ ) as a function of $n_{z}$ is shown in Fig. 2a, as a curve with cross symbols, when $\beta=0.02$. The qualitative feature of Fig. 2a can be understood by looking for steady solutions of Eq. (6) assuming $\mathbf{u}=0$, and a fixed orientation of the ellipsoids. The settling velocity becomes smaller when the value of $n_{z}$ increases, which is a consequence of the increased resistance of the fluid as the largest sides of the ellipsoids become closer to horizontal.

It has already been documented that turbulence may change the settling velocity of droplets in a turbulent flow, see e.g. [36]. Figure. 2a shows the dependence of the settling velocity, $U_{\text {sett }}=-\left\langle v_{z}\right\rangle$, conditioned on the angle $n_{z}$, for the three Reynolds numbers studied here. Remarkably, as the Reynolds number is increased, the difference between the fastest and the slowest ellipsoids increases. Namely, the settling velocity of the ellipsoids, at small values of $n_{z}$, significantly increases. On the other hand, the slowest settling velocities, when $n_{z} \approx 1$, decrease when $R_{\lambda}$ increases. Averaged over all possible directions, the settling velocity $U_{\text {sett }}$ shows a weak variation as a function of the Reynolds number: it is $\approx 9.9 \mathrm{~cm} / \mathrm{s}$ for $R_{\lambda}=56$ and $R_{\lambda}=95$, and $\approx 10.4 \mathrm{~cm} / \mathrm{s}$ for $R_{\lambda}=151$. Overall, these slight variations of 
$U_{\text {sett }}$ are consistent with those reported in [26]. As expected, the fluctuations of the settling velocity increase when the energy dissipation, $\varepsilon$, increases. Fig. 2(a) implies that two particles very close to each other may have a very significant velocity difference, provided the two ellipsoids have a different orientation. This effect becomes stronger when $R_{\lambda}$ increases. In a suspension of identical particles, in the absence of gravity, the velocity differences between particles close to each other is much smaller. This difference is at the origin of the very different collision rates obtained in the presence and in the absence of gravity, reported in the following section.

Completely similar effects are also observed at different values of $\beta$. For the ellipsoids considered here, with a fixed value of $a$, the main effect of $\beta$ in the range considered here, $0.01 \leq \beta \leq 0.05$, is to increase the mass of the particles, leaving almost unchanged the tensor $\hat{\mathbf{K}}$ defined in Eq. (4). This immediately leads to the conclusion that the settling velocity should grow essentially linearly with $\beta$ for the range of values considered, consistent with the values indicated in Table II.

On general grounds, the variance of the velocity fluctuations of the flow, $\left\langle\mathbf{u}^{2}\right\rangle$ is related to the dissipation, $\varepsilon$, and the integral length scale of the flow, $L$, as:

$$
\left\langle\mathbf{u}^{2}\right\rangle \propto(\varepsilon L)^{2 / 3} .
$$

This relation implies that the ratio between the settling velocity, which does not vary much with the Reynolds number, and the root mean square $(r m s)$ of the velocity fluctuations should roughly go as $\propto \varepsilon^{-1 / 3}$ (note that in the case of spherical particles at Stokes numbers comparable to those considered here, the variance of the velocity of the fluid and of the particles are very close to each other [37]). This expectation is consistent with our own numerical findings, see Fig. 2(b) (the size of the integral length scale of the velocity here is $\approx 5 \mathrm{~cm}$ ). The range of values of $\varepsilon$ considered here covers cases where the settling velocity is larger than the rms of the turbulent velocity fluctuations $\left(\varepsilon \approx 1 \mathrm{~cm}^{2} / \mathrm{s}^{3}\right.$; $\left.R_{\lambda}=56\right)$, where the two velocities are comparable $\left(\varepsilon \approx 16 \mathrm{~cm}^{2} / \mathrm{s}^{3} ; R_{\lambda}=95\right)$, and where the turbulent fluctuations dominate $\left(\varepsilon \approx 256 \mathrm{~cm}^{2} / \mathrm{s}^{3} ; R_{\lambda}=151\right)$. Based on our own numerical results in turbulent flows with $\varepsilon \approx 16 \mathrm{~cm}^{2} / \mathrm{s}^{3}$ $\left(R_{\lambda} \approx 95\right)$, the picture is completely similar for the other values of $\beta$.

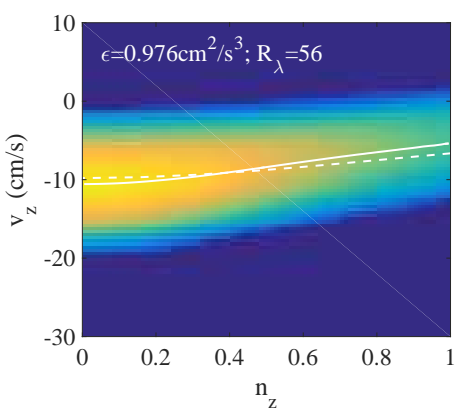

(a)

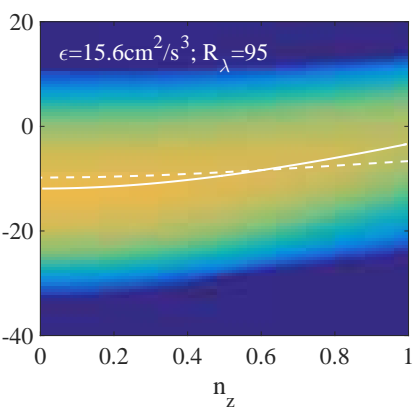

(b)

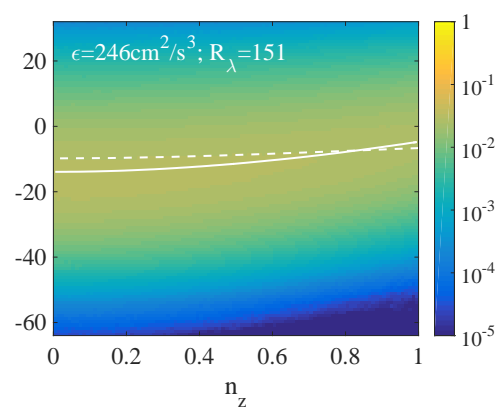

(c)

FIG. 3. Comparison between velocity fluctuations and settling velocity at different turbulence intensity. The figure shows the joint PDFs of the orientation, $n_{z}$ (horizontal) and of the vertical velocity, $v_{z}$ (vertical) for ellipsoids at $\beta=0.02$, at the three values of $\varepsilon$, as indicated on the figures. The probability is color coded, as indicated on the color bar. The conditional average $\left\langle v_{z} \mid n_{z}\right\rangle$ is indicated by the full line in the figure (the dashed line shows the settling velocity in still fluid).

Further insight on the fluctuations of the settling velocity, and on its dependence on the orientation, $n_{z}$, is provided by Fig. 3. The figure shows the joint PDF of $v_{z}$ (the vertical velocity) and $n_{z}$ for ellipsoids of aspect ratio $\beta=0.02$ at the three turbulence intensities considered. The conditional value of $v_{z}$ on $n_{z},\left\langle v_{z} \mid n_{z}\right\rangle$ is shown as a full line. The vertical extent of the region with a large probability is, up to a numerical prefactor, the rms of the velocity fluctuations. At the smallest Reynolds number, $R_{\lambda}=56$, see the left panel of Fig. 3, the fluctuations in the vertical velocities are small, compared to $\left\langle v_{z} \mid n_{z}\right\rangle$. In particular, the vertical velocity is almost always negative, implying that almost all particles move downward, in the direction imposed by gravity. In contrast, at much larger turbulence intensity, see the right panel of Fig. 3, the velocity fluctuations are large compared to $\left\langle v_{z} \mid n_{z}\right\rangle$, which implies that while ellipsoids move downward, on the mean, they have also large probabilities of moving up. The intermediate case, $R_{\lambda}=95$, shown in the middle panel of Fig. 3, corresponds to a case where ellipsoids can, with a relatively small probability, move up in the gravity field.

Fig. 3 clearly shows that, as expected, the importance of the bias due to settling diminishes when the turbulence intensity, $\varepsilon$, increases. Although the Froude number corresponding to ellipsoids of aspect ratios $\beta=0.02$ at the highest turbulence intensity considered $\left(\varepsilon \approx 256 \mathrm{~cm}^{2} / s^{3}\right)$, is as high as $F r^{s} \approx 1.23$, see Table II, the bias induced by 
settling is actually relatively small. Consistent with this observation, the results presented in Section IV show that the collision rates at $\varepsilon \approx 256 \mathrm{~cm}^{2} / \mathrm{s}^{3}$ with and without gravitational settling do not differ very much.

As pointed out in [18], the proper Froude number to parametrize the bias is rather $F r^{L}$, defined by Eq. (10). Cases corresponding to $\mathrm{Fr}^{L}<1$ correspond to an almost uniform distribution of $n_{z}$.

\section{COLLISIONS}

\section{A. Definition of the collision rates}

The probability of collisions between identical particles can be conveniently expressed in terms of the collision kernel, $K$, defined by expressing the number of collisions $N_{c}$ over a time $T$, in a volume $V$ containing $N$ particles $(N \gg 1)$ as:

$$
N_{c}=\frac{1}{2} K \times \frac{N^{2}}{V} \times T \text {. }
$$

The collision kernel has therefore the dimension of the cube of a length (a volume) divided by a time. It depends on the Reynolds number, the shape of the particles, and on the gravity acceleration.

Over the years, a good understanding of the collision rate in a turbulent, monodisperse suspension of spherical particles has been obtained. The pioneering work of Saffman and Turner [38] provides an explicit expression for the collision rate in the case of spherical droplets which exactly follow the flow. In the case of inertial particles, the collision rate is enhanced due to, on the one hand, an enhanced concentration of particles around existing particles in the suspension [39], and on the other hand, a much increased relative velocity of the particles when they collide [40, 41]. Numerical studies indicate that the latter effect prevails in determining the collision rate in a turbulent suspension as soon as the inertia of the particles is significant [12, 42].

Interestingly, several simulations have pointed that at a fixed turbulence rate, the effect of gravitational settling is to reduce the collision rate of spherical particles $[13,14,43]$.

\section{B. Collision rate statistics}

The collision kernel, $K$, expressed in $\mathrm{cm}^{3} / \mathrm{s}$ and measured from our own DNS, is shown in Fig. 4 as a function of the dissipation rate $\varepsilon$ in the fluid, both in the presence and in the absence of gravitational settling.

The collision rate is first shown as a function of $\beta$, at the intermediate value of $\varepsilon \approx 16 \mathrm{~cm}^{3} / \mathrm{s}$, see Fig. 4a. The collision rate is much smaller in the absence, than in the presence of gravity. Figure 4a indicates an increase, essentially linear, of the collision rate as a function of $\beta$.

Figure. $4 \mathrm{~b}$ shows the collision rate as a function of the energy dissipation, $\varepsilon$, and demonstrates once again that at small Reynolds numbers, the collision kernel is vastly enhanced by the presence of gravity. It is, at the smallest Reynolds numbers considered here $\left(\varepsilon \approx 1 \mathrm{~cm}^{2} / \mathrm{s}^{3}\right), \sim 40$ times superior in the presence of gravity, than without it. The ratio however significantly drops when increasing the Reynolds number, and at the highest Reynolds number here $\left(\varepsilon \approx 256 \mathrm{~cm}^{2} / \mathrm{s}^{3}\right)$, the ratio is only slightly larger than 1 .

In Section IV D we will discuss the physical mechanisms possibly responsible for the behavior shown in Fig. 4.

\section{Orientation of colliding ellipsoids in the presence of gravity}

As already explained, see Section III B, the dependence of the settling velocity on the angle between the ellipsoid and the direction of gravity naturally provides a dispersion of the relative velocity of the particles close to each other, thus potentially enhancing the collision rate.

To explore further these issues, Fig. 5a-c show the orientation of two colliding ellipsoids, at the three Reynolds numbers considered. The continuous line shows the PDF of the orientations of all the ellipsoids. We distinguish the two colliding ellipsoids by identifying the one which has, at the time of the collision, the highest position of its center of mass. The distribution of the angles $n_{z}$ for the upper ellipsoid is shown as a dashed line, whereas the distribution for the lower ellipsoid is shown as a dashed-dotted line. Overall, Fig. 5 shows that the higher ellipsoid tends to have predominantly a smaller value of $n_{z}$ than the lower ellipsoid, whose probability of orientation has more weight at larger values of $n_{z}$. This tendency persists for all the values of $\varepsilon$ considered in this study. For the two largest values of $R_{\lambda}$ considered, the probability of orientation of the lower ellipsoids has even more weight at larger values of $n_{z}$ than at smaller ones. 


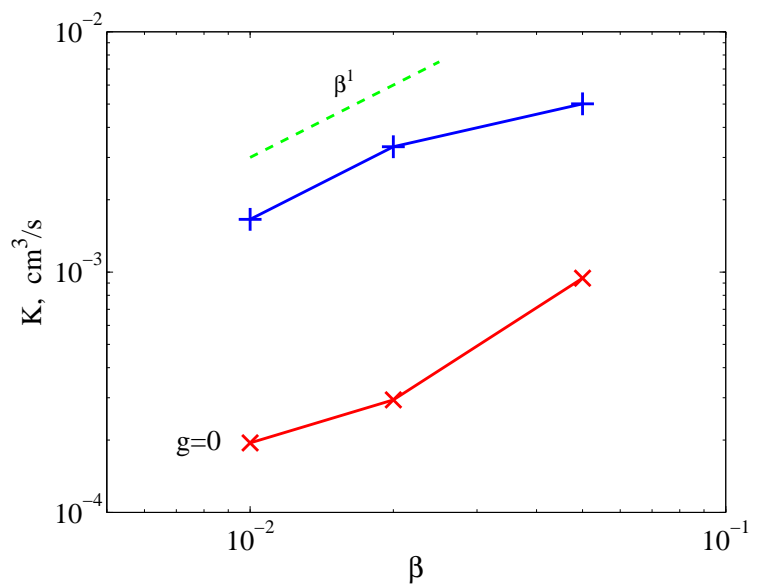

(a)

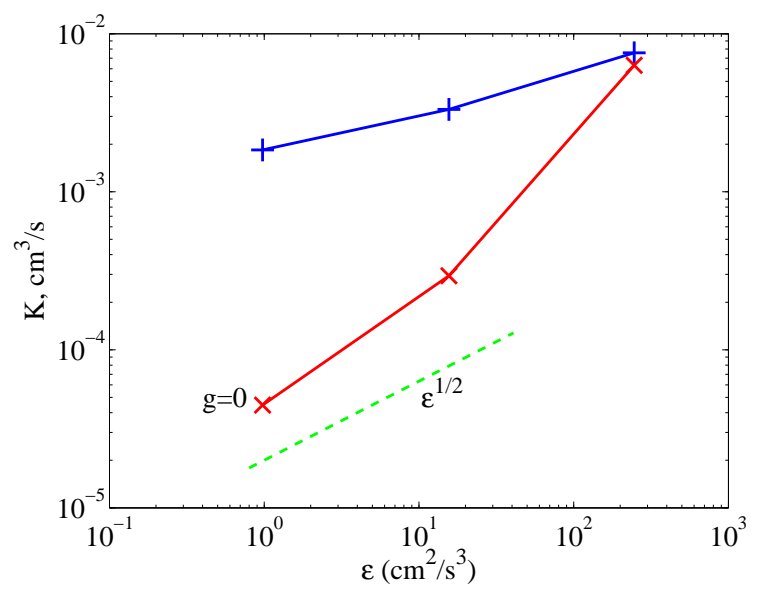

(b)

FIG. 4. Collision rate of a suspension of ellipsoids. The collision rate in the presence ("+" symbols) and in the absence (" $\times$ " symbols) of gravitational settling are compared. Panel (a) Collision rate as a function of $\beta$, at a fixed Reynolds number, $R_{\lambda}=95$ $\left(\varepsilon \approx 16 \mathrm{~cm}^{2} / \mathrm{s}^{3}\right)$. The collision rate increases slightly with $\beta$ in both cases, and is much larger in the presence of gravity. Panel (b) Collision rate as a function of $\varepsilon$, at a fixed value of $\beta=0.02$. Generally, the collision rates in the absence of gravity (shown with a " $\times$ " symbol) are much smaller than in the presence of gravity (shown with a "+" symbol). The difference however, diminishes when the Reynolds number increases.

In comparison, Fig. 6 shows the distribution of the relative angle between the two colliding ellipsoids, both in the presence and in the absence of gravitational settling. Specifically, Fig. 6 shows the cumulative PDF, defined as:

$$
\Pi(\xi)=\int_{0}^{\xi} P\left(\xi^{\prime}\right) d \xi^{\prime}
$$

where $P(\xi)$ is the PDF of the cosine of the angle $\left(\mathbf{n}_{1} \cdot \mathbf{n}_{2}\right)$ between the vectors $\mathbf{n}_{1,2}$ characterizing the orientations of the two ellipsoids. In all cases, Fig. 6 shows a sharp increase of $\Pi$ close to $\mathbf{n}_{1} \cdot \mathbf{n}_{2}=1$, that is, for perfectly aligned ellipsoids (the $\operatorname{PDF} P(\xi)$ shows a very narrow peak near $\xi=1$ ). This corresponds to a very high probability of the two ellipsoids colliding while perfectly aligned with each other. The probability of the two ellipsoids colliding at a finite angle is clearly larger in the case with gravity than in the case without gravity. This is consistent with the fact that in the presence of gravity, many collisions occur due to a differential gravitational settling. While this difference is visible at each of the three Reynolds numbers investigated, Fig. 6 shows that the difference becomes weaker at the highest Reynolds number studied. This is consistent with the fact that, as the effect of turbulence becomes stronger compared to that of gravitational settling, the difference in the nature of the collisions between the two cases diminishes.

\section{Discussion}

To provide an explanation of the dependence of the collision rate $K$ on $\varepsilon$ and $\beta$ shown in Fig. 4 , we begin by recalling that the collision kernel can be interpreted as a flux of particles incoming into one of them. While this flux can be explicitly computed in the case of colliding spheres, an exact determination of the collision rate is much more difficult in the problem of colliding ellipsoids considered here. Still, as we show in this subsection, the interpretation in terms of a flux allows us to provide qualitative estimates, as well as a qualitative explanation of the trends observed in Sections IV B and IV C.

\section{Influence of gravitational settling}

Consider first the case of ellipsoids in the presence of gravitational settling. As stressed in Section III B, gravitational settling induces a strong relative velocity difference between ellipsoids with a different orientation, see Fig. 2. Based on 


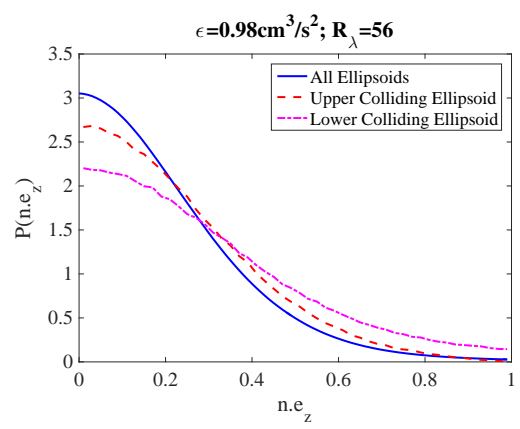

(a)

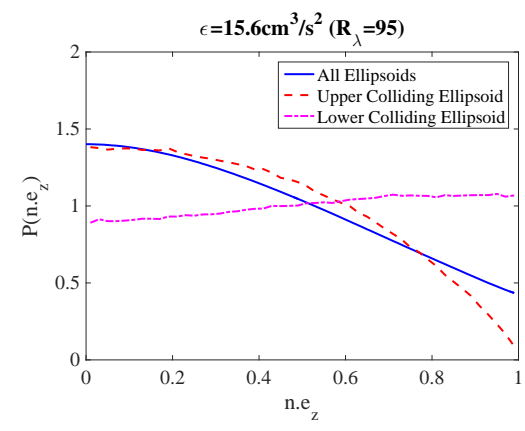

(b)

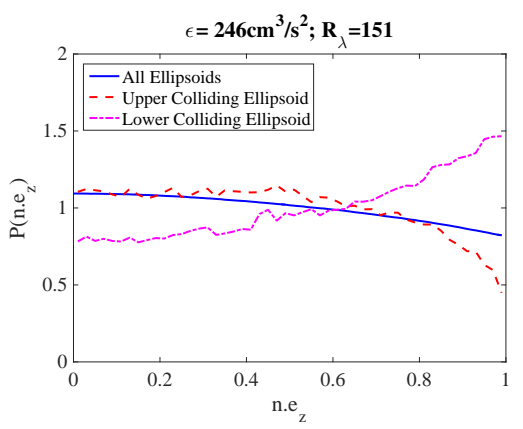

(c)

FIG. 5. Distribution of $n_{z}$ for the colliding ellipsoids during their collision: upper ellipsoid (dashed red line), lower ellipsoid (dashed-dotted magenta line). As a comparison, the distribution of the angles for all ellipsoids is shown with a full line. The Reynolds number increases from $R_{\lambda}=56$ (left), $R_{\lambda}=95$ (center) and $R_{\lambda}=151$ (right). The results are shown for ellipsoids with $\beta=0.02$. The upper (lower) ellipsoids have a larger probability of having a small (large) value of $n_{z}$, which implies that the upper ellipsoids settle faster than the lower ones.

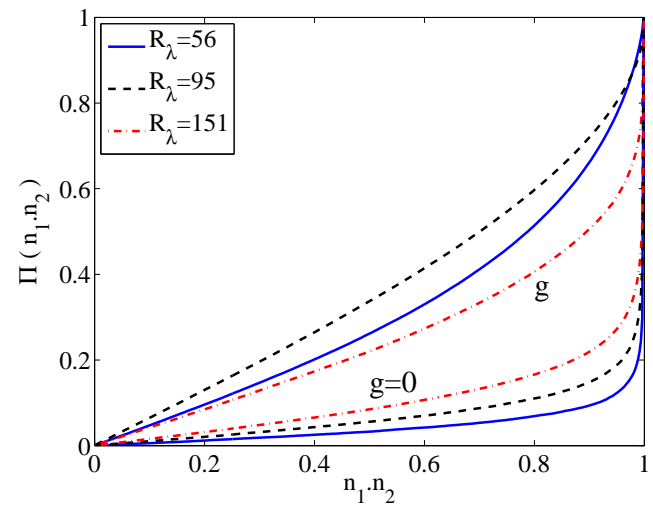

FIG. 6. Cumulative probability distribution function of the cosine of the relative angle between two colliding ellipsoids, $\mathbf{n}_{1} \cdot \mathbf{n}_{2}$. The full, dashed, and dashed-dotted lines correspond to $R_{\lambda}=56, R_{\lambda}=95$ and $R_{\lambda}=151$, respectively. The three lowest (respectively highest) curves correspond to a case without (respectively with) gravitational settling. The cumulative probability distribution function is defined by Eq. 13.

Eq. (6), the velocity difference is expected to be proportional to $2 \beta g \tau_{S p}$. The values shown in Fig. 2 suggest a value of the velocity differences $\Delta u_{s} \approx \beta g \tau_{S p} / 5$, which leads to $\Delta u_{s} \approx 1.1 \mathrm{~cm} / \mathrm{s}$. The flux of particles incoming into an oblate ellipsoid can be estimated as the product of the area of its largest side, $\sim \pi a^{2}$, and of the relative velocity between two objects. This provides, as an estimate of the collision rate of the particles, $K \approx \frac{\pi}{5} a^{2} \beta g \tau_{S p}$. In the case of ellipsoids with $\beta=0.02$ and $\varepsilon \approx 16 \mathrm{~cm}^{2} / \mathrm{s}^{3}$, this leads to $K \approx 810^{-4} \mathrm{~cm}^{3} / \mathrm{s}$, which provides the right order of magnitude for the value of $K$ determined numerically, $K \approx 3.310^{-4} \mathrm{~cm}^{3} / \mathrm{s}$ (see Fig. $4 \mathrm{~b}$ ). The estimate above for the collision rate also predicts a growth of $K \propto \beta$. This is certainly consistent with the trend observed at $\varepsilon \approx 16 \mathrm{~cm}^{2} / \mathrm{s}^{3}$, between $\beta=0.01$ and $\beta=0.02$. The growth of $K$ is slower between $\beta=0.02$ and $\beta=0.05$, which can be understood as a consequence of the increased importance of inertia, as explained below. Last, the estimate above does not involve the intensity of turbulence, $\varepsilon$. This is qualitatively consistent with the relatively weak increase of $K$, when $\beta=0.02$, as $\varepsilon$ increases from $1 \mathrm{~cm}^{2} / \mathrm{s}^{3}$ to $256 \mathrm{~cm}^{2} / \mathrm{s}^{3}$.

\section{Influence of turbulence in the absence of gravitational settling}

We now turn to estimating the collision rate in the absence of gravitational settling, and begin with the case where the motion of the ellipsoids can be thought of as a simple advection by the flow. This picture is expected to be correct 
when the relaxation time of the particles, is very short compared to the time of the turbulent eddies i.e. when the Stokes number $S t$ is very small. In this case, the collision between particles is due to the relative motion between two ellipsoids, which in turn results from the velocity gradients. Specifically, two objects separated by a distance $a$, small compared to the Kolmogorov length, have a relative velocity $\propto a / \tau_{K}[12,38]$, where $\tau_{K}=(\nu / \varepsilon)^{1 / 2}$ is the Kolmogorov time. This leads to an estimate of $K \approx \pi a^{3}(\varepsilon / \nu)^{1 / 2}$. The corresponding numerical values are $K \approx 3 \times 10^{-5} \mathrm{~cm}^{3} / \mathrm{s}$ when $\varepsilon \approx 1 \mathrm{~cm}^{2} / \mathrm{s}^{3}$ and $K \approx 1.2 \times 10^{-4} \mathrm{~cm}^{3} / \mathrm{s}$ when $\varepsilon \approx 16 \mathrm{~cm}^{2} / \mathrm{s}^{3}$. These values only slightly understimate the collision rate determined numerically in the absence of gravity, see the lower curve of Fig. 4b. Our estimate also predicts a dependence of $K \propto \varepsilon^{1 / 2}$ (all the other parameters being fixed). This is close to the dependence observed in the range $1 \mathrm{~cm}^{2} / \mathrm{s}^{3} \leq \varepsilon \leq 16 \mathrm{~cm}^{2} / \mathrm{s}^{3}$. In sharp contrast, this estimate is inappropriate to explain the collision rate at $\varepsilon \approx 256 \mathrm{~cm}^{2} / \mathrm{s}^{3}$ and the fact that, in the absence of gravitational settling, the collision kernel $K$ grows much faster than $K \propto \varepsilon^{1 / 2}$ in the range $16 \mathrm{~cm}^{2} / \mathrm{s}^{3} \lesssim \varepsilon \lesssim 256 \mathrm{~cm}^{2} / \mathrm{s}^{3}$, see Fig. $4 \mathrm{~b}$.

We note that the estimates for $K$, with and without gravitational settling, obtained by neglecting the particles inertia, make possible a direct comparison between the effects of differential settling, and the effect of turbulence. Determining when $K \approx \frac{\pi}{5} a^{2} \beta g \tau_{S p}$ and $K \approx a^{3} / \tau_{K}$ become comparable leads to the conclusion that the effect of gravitational settling should become negligible compared to that of turbulence when $a / \tau_{K} \gtrsim \beta g \tau_{S p}$, or equivalently, $\varepsilon \gtrsim \nu\left(\beta g \tau_{S p} / a\right)^{2}$. With the parameter value $\beta=0.02$, this points to a value of $\varepsilon \approx 500 \mathrm{~cm}^{2} / \mathrm{s}^{3}$.

As we now argue, this argument overestimates the value of $\varepsilon$ at which turbulence becomes dominant, as inertial effects have been so far completely ignored.

\section{Inertial effects}

The strong deviation from the qualitative prediction that $K \approx a^{3} / \tau_{K}$ actually points to the importance of inertial effects. In turbulent suspensions of spherical droplets, similar deviations are known to occur when the inertia of the particles, measured by the Stokes number, Eq. (8), increases [12, 39-41]. The very substantial increase of the collision rate can be attributed either to the effect of the preferential concentration of droplets in certain regions of the flow, or to the increased relative velocity between close particles (the "sling effect" [14, 40]). Careful numerical results establish that the latter effect plays the dominant role in the increase of the collision rate [42], as soon as the Stokes number of the particles $S t=\tau_{S p} / \tau_{K} \gtrsim 0.5$, where $\tau_{S p}=2 / 9\left(r^{2} / \nu\right)\left(\rho_{p} / \rho_{f}\right)$, where $a$ is the radius of the spheres.

As indicated in Table II, the Stokes number of the particles considered in the present study is of order 0.5 at the highest value of $\varepsilon$ simulated here. The results concerning collisions in droplet suspensions then provide an explanation for the very sharp increase of $K$ for particles with $\beta=0.02$, between $\varepsilon \approx 16 \mathrm{~cm}^{2} / \mathrm{s}^{3}(S t \approx 0.135)$ and $\varepsilon \approx 256 \mathrm{~cm} / \mathrm{s}^{3}$ $(S t=0.54)$. We also note that the Stokes number for particles with $\beta=0.05$ and $\varepsilon \approx 16 \mathrm{~cm}^{2} / \mathrm{s}^{3}$ is $S t \approx 0.34$, which points to significant effects of particle inertia, and could explain the significant difference between the collision rates $K$ between $\beta=0.02$ and $\beta=0.05$, in the absence of gravity. It may also explain the departure from the linear dependence on $\beta$, in the presence of gravity, when $\beta$ increases to 0.5 , as the effects of turbulence become important. Distinguishing quantitatively the effect of differential settling from that of inertia in this case, however, is likely to be a challenging task.

\section{CONCLUSIONS}

In this work, we have considered the collision of oblate ellipsoids, of properties close to those of ice crystals, in a turbulent flow whose intensity ranges from $\sim 1 \mathrm{~cm}^{2} / \mathrm{s}^{3}$ to $\approx 256 \mathrm{~cm}^{2} / \mathrm{s}^{3}$. The parameters chosen here are close to those observed in moderately turbulent clouds. The motion of the ellipsoids is determined numerically by solving the equations determining their translation and rotation. These equations use the expressions of the force and torque derived in the limit where the Reynolds number of the flow around the object is very small. The collision rate has been determined using the ghost-particle approximation, i.e., by following independent trajectories of ellipsoids, and determining when they collide in the flow.

In the presence of gravity, our results show that crystals tend to settle edge first. We also observed a strong variation of the settling velocity with the orientation, qualitatively consistent with the observed gravitational settling in still air. Over the range of studied turbulence intensity, this effect tends to increase with $\varepsilon$. On the other hand, the alignment effect of the ellipsoid with gravity, $\mathbf{g}$, tends to diminish when the intensity of turbulence increases. This can be attributed to an associated increase in the turbulent stress acting on the ellipsoid.

Our study points to the competition between several physical effects which strongly influence the collision rate. As pointed out already [15], differential gravitational settling plays a very important role, in particular at very small Reynolds numbers $\left(\varepsilon \approx 1 \mathrm{~cm}^{2} / \mathrm{s}^{3}\right)$. In this regime, a direct comparison between collision rate with and without gravitational settling reveals that the collision rate is reduced by almost two orders of magnitude in the absence of 
g. As $\varepsilon$ increases, however, the collision rate induced in the turbulent flow without gravitational settling increases very rapidly, so the relative influence of $\mathbf{g}$ rapidly decreases: at $\varepsilon \approx 256 \mathrm{~cm}^{2} / \mathrm{s}^{3}$, the collision rates with and without $\mathrm{g}$ are very close. Thus, the very strong effect of gravitational settling seems to be limited to small Reynolds numbers. Although the role of gravitational settling becomes weaker when the turbulence intensity increases, the analysis of the relative orientation between two colliding ellipsoids reveals that the influence of gravity remains significant, even at the highest value of $\varepsilon$ considered here.

It is not completely clear whether at still higher values of the turbulence intensity, gravitational settling will further increase the collision kernel, as it is the case at low Reynolds numbers, or whether it will in fact lower the collision rate, as it happens for droplets.

The model used here, based on a set of equations which do not take into account any inertia correction, predicts in agreement with previous work $[18,26]$ a tendency of the ellipsoids to settle with their edges first. This clearly differs from the expectation based on the torque due to inertia corrections [19, 24]. Qualitatively, the conclusion reached in this work, namely that the collision rate between crystals is due to differential settling at small Reynolds number, and to turbulence at higher Reynolds number, is very unlikely to depend very sensitively on the precise nature of the distribution of the particles.

Geometrical technicalities make a detailed analytical calculation of the collision rate difficult, thus preventing an accurate estimate in the case where particles follow the flow, as done in the case of droplets [38]. Simple estimates however can provide satisfactory predictions for the collision kernel, both in the presence and in the absence of gravitational settling, at least when the response time of the particles is sufficiently small compared to the smallest characteristic time in the flow, the Kolmogorov time. As already known for droplets, we find an important enhancement of the collision kernel for ellipsoids when particle relaxation time becomes large compared to the Kolmogorov time scale.

Acknowledgements: We are thankful to S. Borrmann, K. Gustavsson, S. Malinowski, B. Mehlig, R. Shaw and C. Siewert for discussions at various stages of the work. The simulations have been performed by using the local HPC facilities at ENS de Lyon (PSMN) and at the university Lyon-I (P2CHPD) mainly supported by the Auvergne-RhôneAlpes region (GRANT CPRT07-13 CIRA) and the project Equip@Meso (ANR-10-EQPX-29-01). AN, EL and AP have been supported by the grant "TEC" 2 from A.N.R. (ANR-12-BS09-0011-02), and by the EU COST action MP0806 "Particles in Turbulence". JJ and AP acknowledge the support from the Alexander-von-Humboldt foundation.

[1] H. R. Pruppacher and J. D. Klett, Microphysics of clouds and precipitations (Springer, Dordrecht, Heidelberg, London, New York, 2010).

[2] P. Yang, H. Wei, H. L. Huang, B. A. Baum, Y. X. Hu, G. W. Kattawar, M. I. Mischenko, and Q. Fu, "Scattering and absorption property database for nonspherical ice particles in the near-through far-infrared spectral region," Appl. Opt. 44, 32-63 (2005).

[3] J. Um, G. M. McFarquhar, Y.P. Hong, S.-S. Lee, C. H. Jung, R. P. Lawson, and Q. Mo, "Dimensions and aspect ratios of natural ice crystals," Atmos. Chem Phys. 15, 3933-2956 (2015).

[4] P. Yang, K. N. Liou, L. Bin, B. Yi, and B. A. Baum, "On the radiative properties of ice clouds: light scattering, remote sensing and radiation parametrization," Adv. Atmos. Sci. 32, 32-63 (2015).

[5] R. A. Shaw, "Particle-turbulence interactions in atmospheric clouds," Ann. Rev. Fluid Mech. 35, 183-227 (2003).

[6] E. Bodenschatz, S. P. Malinowski, R. A. Shaw, and F. Stratmann, "Can we understand clouds without turbulence ?" Science 327, 970-971 (2010).

[7] W. W. Grabowski and L. P. Wang, "Growth of cloud droplets in a turbulent environment," Ann. Rev. Fluid Mech. 45, 293 (2013).

[8] J. P. Chen and D. Lamb, "The theoretical basis for the paramterization of ice crystal habits: growth by vapor deposition," J. Atmos. Sci. 51, 1206-1221 (1994).

[9] T. Hashino, K. Y. Cheng, C. C. Chueh, and P. K. Wang, "Numerical study of motion and stability of falling columnar crystals," J. Atmos. Sci. 73, 1923 (2016).

[10] F. Yang, M. Ovchinnikov, and R. A. Shaw, "Minimalist model of ice microphysics in mixed-phase stratiform clouds," Geophys. Res. Lett. 40, 3756-3760 (2013).

[11] F. Yang, M. Ovchinnikov, and R. A. Shaw, "Microphysical consequences of the spatial distribution of ice nucleation in mixed-phase stratiform clouds," Geophys. Res. Lett. 41, 5280-5287 (2014).

[12] A. Pumir and M. Wilkinson, "Collisional aggregation due to turbulence," Ann. Rev. Condens. Matter Phys. 7, 141-170 (2016).

[13] W. Grabowski and P. Vaillancourt, "Comments on "preferential concentration of cloud droplets by turbulence: effects on the early evolution of cumulus cloud droplet spectra"," J. Atmos. Sci. 56, 1433 (1999).

[14] G. Falkovich and A. Pumir, "Sling effect in collisions of water droplets in turbulent clouds," J. Atmos. Sci. 64, 4497 (2007). 
[15] C. Siewert, R. P. J. Kunnen, and W. Schröder, "Collision rates of small ellipsoids settling in turbulence," J. Fluid Mech. 758, 686-701 (2014).

[16] G. B. Jeffery, "The motion of ellipsoid particles immersed in a viscous fluid," Proc. Roy. Soc. A 102, 161-179 (1922).

[17] J. Happel and H. Brenner, Low Reynolds Number Hydrodynamics (Martinus Nijhoff Publishers, The Hague, The Netherlands, 1983).

[18] K. Gustavsson, J. Jucha, A. Naso, E. Lévêque, A. Pumir, and B. Mehlig, "Statistical model for the orientation of a non-spherical particle settling in turbulence," preprint (2017).

[19] R. G. Cox, "The steady motion of particles of arbitrary shape at small reynolds numbers," J. Fluid Mech. 23, 625-643 (1965).

[20] W. Chester, "A general theory for the motion of a body through a fluid at low reynolds number," Proc. R. Soc. Lond. A 430, 89-104 (1990).

[21] L. G. Leal, "Particle motions in a viscous fluid," Ann. Rev. Fluid Mech. 12, 435-476 (1980).

[22] F. Candelier, J. Einarsson, and B. Mehlig, "Angular dynamics of a small particle in turbulence," Phys. Rev. Lett. 117, $204501(2016)$.

[23] D. Lopez and E. Guazzelli, "Inertial effects on fibers settling in a vortical flow," Phys. Rev. Fluids 2, 024306 (2017).

[24] J. D. Klett, "Orientation model for particles in turbulence," J. Atmos. Sci. 52, 2276-2285 (1995).

[25] M. Voßkuhle, E. Lévèque, M. Wilkinson, and A. Pumir, "Multiple collisions in turbulent flows," Phys. Rev. E 88, 063008 (2013).

[26] C. Siewert, R. P. J. Kunnen, and W. Schröder, "Orientation statistics and settling velocity of ellipsoids in decaying turbulence," Atmos. Res. 142, 45-56 (2014).

[27] Y. K. Choi, J. W. Chang, W. Wang, M.-S. Kim, and G Elber, "Continuous collision for ellipsoids," IEEE Trans. Vis. Comput. Graphics 15, 311-325 (2009).

[28] K. M. Markowicz and M. L. Witek, "Simulations of contrail optical properties and radiative forcing for various crystal shapes," J. Appl. Meteor. Climatol. 50, 1740-1755 (2011).

[29] K. Gustavsson and B. Mehlig, "Statistical models for spatial patterns of heavy particles in turbulence," Adv. Phys. 65, 1 (2016).

[30] M Shin and D. L. Koch, "Rotational and translational dispesion of fibers in isotropic turbulent flows," J. Fluid Mech. 540, 143-173 (2005).

[31] A. Pumir and M. Wilkinson, "Orientation statistics of small particles in turbulence," New J. Phys. 13, 093030 (2011).

[32] S. Parsa, E. Calzavarini, F. Toschi, and G. A. Voth, "Rotation rate of rods in turbulent fluid flows," Phys. Rev. Lett. 109, 134501 (2012).

[33] L. Chevillard and C. Meneveau, "Orientation dynamics of small, triaxial-ellipsoidal particles in isotropic turbulence," J. Fluid Mech. 737, 571-596 (2013).

[34] K. Gustavsson, J. Einarsson, and B. Mehlig, "Tumbling of small axisymmetric particles in random and turbulent flows," Phys. Rev. Lett. 112, 014501 (2014).

[35] G. A. Voth and A. Soldati, "Anisotropic particles in turbulence," Ann. Rev. Fluid Mech. 49, $249-276$ (2017).

[36] G. H. Good, P. J. Ireland, G. P. Bewley, E. Bodenschatz, L. R. Collins, and Z. Warhaft, "Settling regimes of inertial particles in isotropic turbulence," J. Fluid Mech. 759, R3 (2014).

[37] M. Gibert, H. Xu, and E. Bodenschatz, "Where do small weakly inertial particles go in a turbulent flow ?" J. Fluid Mech. 698, 160-167 (2012).

[38] P. G. Saffman and J. S. Turner, "On the collision of droplets in turbulent clouds," J. Fluid Mech. 1, 16-30 (1956).

[39] S. Sundaram and L. R. Collins, "Collision statistics in an isotropic particle-laden turbulent suspension. part 1. direct numerical simulations," J. Fluid Mech. 335, 16-30 (1997).

[40] G. Falkovich, A. Fouxon, and M. G. Stepanov, "Acceleration of rain initiation by cloud turbulence," Nature 419, 151 (2002).

[41] M. Wilkinson and B. Mehlig, "Caustics in turbulent aerosols," EPL (Europhysics Letters) 71, 186 (2005).

[42] M. Voßkuhle, A. Pumir, E. Lévêque, and M Wilkinson, "Prevalence of the sling effect for enhancing collision rates in turbulent suspensions," J. Fluid Mech. 749, 841-852 (2014).

[43] O. Ayala, B. Rosa, L. P. Wang, and W. W. Grabowski, "Effects of turbulence on the geometric collision rate of sedimenting droplets. part 1. results from direct numerical simulation," New J. Phys. 10, 075015 (2008). 